Evolutionarily-conserved mechanisms of male germline development in flowering plants and animals

Patrícia A. Pereira1 ${ }^{1}$, Paulo Navarro-Costa ${ }^{1}$, Rui G. Martinho ${ }^{1,2}$ and Jörg D. Becker $1,{ }^{*}$

1Instituto Gulbenkian de Ciência, Rua da Quinta Grande 6, 2780-156 Oeiras, Portugal

${ }^{2}$ Regenerative Medicine Program, Departamento de Ciências Biomédicas e Medicina, and IBB-Institute for Biotechnology and Bioengineering, Centro de Biomedicina Molecular e Estrutural, Universidade do Algarve, Campus de Gambelas, 8005-139 Faro, Portugal

*Corresponding Author: Jörg D. Becker; email: jbecker@igc.gulbenkian.pt 


\title{
Evolutionarily-conserved mechanisms of male germline development in flowering plants and animals
}

\begin{abstract}
Sexual reproduction is the main reproductive strategy of the overwhelming majority of Eukaryotes. This suggests that the last Eukaryotic common ancestor was able to reproduce sexually. Sexual reproduction reflects the ability to perform meiosis, and ultimately generating gametes, which are cells that carry recombined half sets of the parental genome and are able to fertilize. These functions have been allocated to a highly specialized cell lineage: the germline. Given its significant evolutionary conservation, it is to be expected that the germline program shares common molecular bases across extremely divergent Eukaryotic species. In this review we aim to identify the unifying principles of male germline establishment and development by comparing two very disparate kingdoms: plants and animals. We argue that male meiosis defines two temporally-regulated gene expression programs: the first required for meiotic commitment, the second for the acquisition of fertilizing ability. Small RNA pathways are a further key communality, ultimately ensuring the epigenetic stability of the information conveyed by the male germline.
\end{abstract}

Key words: male germline, gametogenesis, flowering plants, animals, meiosis, small RNAs.

Abbreviations: PPC, primary parietal cell; PSC, primary sporogenous cell; PMC, pollen mother cell; PMI, pollen mitosis I; PMII, pollen mitosis II; SCs, sperm cells; MGU, male germ unit; VN, vegetative nucleus; MGH3, MALE GAMETE-SPECIFIC HISTONE H3; HAP2(GCS1), HAPLESS2 (GENERATIVE CELL-SPECIFIC PROTEIN 1); DUO1, DUO POLLEN1; SSP, SHORT SUSPENSOR; piRNAs, piwi-interacting RNAs; miRNAs, microRNAs; siRNAs, short interfering RNAs; DCL1, DICER-LIKE1; TEs, transposable elements.

Male germline establishment and development

The life cycle of plants alternates between two morphologically distinct generations: the sporophyte and the gametophytes. During the course of evolution the sporophytic phase became dominant, with the short-lived gametophytes arising, at the end of ontogeny, within structures on the sporophyte. It is during the development of the gametophytes that the plant germline is specified. Animals also display considerable variation in the timing of germline specification: this lineage can be established during early or late embryogenesis or even throughout adult life, as seen in plants (for a review [1]). Interestingly, in the basal animal lineages, germ cells arise from precursors also capable of generating somatic cell types. This fluid boundary between germline and soma finds parallel with what observed in plants, suggesting the conservation of key developmental mechanisms.

In flowering plants, male gametophytes are formed in specialized reproductive organs of the flower, the anthers. Archesporial cells in the stamen primordium divide periclinally to produce an outer primary parietal cell (PPC) and an inner 
primary sporogenous cell (PSC) (Figure 1). The PPC differentiates into the tapetum - a sterile nourishing tissue essential for the early stages of pollen development. Consecutive divisions of the PSC generate a central mass of microsporocytes, the pollen mother cells (PMCs). PMCs remain interconnected by cytoplasmic bridges - the cytomictic channels. These channels result from the continuity of the inner side of the cytoplasmic membrane (symplast) of neighbouring cells and are believed to be necessary for the subsequent synchronization of PMC development. Such synchronization will regulate meiosis, a process that will ultimately result in the formation of four microspores (for a review [2]). Each microspore will undergo an asymmetric mitotic division, pollen mitosis I (PMI), to form a bicellular structure in which the generative cell (germ cell) is embedded within the cytoplasm of the vegetative cell (pollen grain) via the formation of an endomembrane. This asymmetric division is determinative for the establishment of the plant germline since it results in two daughter cells with distinct fates. More specifically, the generative cell gives rise to the male germline while the vegetative cell (somatic) serves a supporting role in germline development and fertilization. Some isolated mutants show the importance of the asymmetric cell division for proper germline development. In sidecar pollen $(s c p$ ) mutants, microspores divide symmetrically followed by a second asymmetric division of only one of the daughter cells, thus producing mature pollen with an extra vegetative cell [3]. In the case of gemini pollen1 (gem1) mutants, phenotypes ranging from asymmetric to symmetric microspore division can be observed, leading to a variable acquisition of generative cell fate depending on the degree of division asymmetry [4]. Therefore, germ cell fate appears to be dependent on the regulated partitioning of cytoplasmic germline determinants resulting from the asymmetric PMI division [5]. This process finds clear parallels in animals that specify their germline via a preformation mechanism. In such cases, the acquisition of germ cell fate in the developing embryo is the result of an asymmetric distribution of maternally inherited determinants (germ plasm), either before or immediately after fertilization [1]. In angiosperms, after PMI the generative cell undergoes pollen mitosis II (PMII), generating two identical male gametes, the sperm cells (SCs). Structurally, a tricellular pollen grain enfolds the two non-motile SCs in close association with the nucleus of the vegetative cell. This structural assembly is defined as the male germ unit (MGU) [6]. During pollen tube growth, the vegetative nucleus (VN) is positioned close to the tube tip, with the SCs trailing behind. Seeing that defects in MGU organization seem to affect its migration efficiency through the pollen tube [7], the specific intercellular association between the vegetative and sperm cells is considered important for male germline function. In this process, the cytoplasmic projection between the VN and SCs may represent a preferential communication route for possible metabolic exchanges [6]. Tellingly, membrane proteins such as TETRASPANINS 11 and 12 (TET11-12) have been shown to outline this cytoplasmic projection and also the adhesion microdomain between the contiguous sperm cells [8]. After delivery to the female reproductive tissue, one of the SCs will fuse with the egg cell, giving rise to the zygote, while the second SC fuses with the central cell thus initiating development of the endosperm - a nourishing tissue equivalent to the mammalian placenta.

When comparing male gametogenesis in animals with that of plants, the conservation of several major developmental hallmarks becomes clear. Firstly, 
the conservation of mitosis and meiosis as means of ensuring germ cell proliferation and reductive chromosome segregation, respectively. More specifically, prior to meiotic entry, animal male germ cells engage in active mitotic expansion starting from a germline stem cell population. But in contrast to plants, animal post-meiotic cells are unable to proliferate and instead acquire motility via the differentiation of a flagellum or an equivalent structure [9]. Male gamete motility, although present in lower plants, was subsequently lost in most gymnosperms and in all angiosperms. Cytoplasmic continuity between differentiating male germ cells (or their precursors) is another conserved feature when comparing animal with plant male gametogenesis [10]. In animals, such cytoplasmic sharing may be restricted to the initial mitotic divisions or also include the meiotic products [11]. In plants, the symplastic continuity between PMCs regulates meiotic entry and it can be speculated whether the physical contact between the two SCs in the MGU can actually extend to some degree of cytoplasmic sharing. In both plants and animals, this continuity may be related to a developmentally-regulated allocation of limited cytoplasmic resources and/or represent a mean of ensuring concerted cell development. Finally, the need for a structural association between the somatic and germ cell lineages is an equally conserved feature of plant and animal gametogenesis. The conservation of such support might stem from the probable existence of convergent evolution seeing that the last common ancestor to both plants and animals was most likely unicellular. The soma-germline associations may only be patent during the initial regulation of the germline stem cell population or also serve a supporting role throughout all of gametogenesis, as in our species [12]. More specifically, in mammals the somatic Sertoli cells support gametogenic progression by a continual direct contact with developing germ cells. In higher plants the somatic VN supports the fertilizing ability of the enclosed SCs (via pollen tube formation) and is also involved in transposon silencing in the germline (see below). Irrespective of the system, the conservation of the soma-germline associations is likely indicative of a common need of establishing a precise cellular environment for successful male gametogenesis.

Transcriptional programme of male germline development

While the cytology of male germline development has been well characterized both in plants and animals, the actual molecular mechanisms driving this differentiation are less clear. In fact, only recently the male germline gene expression program started to be unveiled. In maize, gene expression profiling of different anther developmental stages identified three distinct gene repertoires. These consisted of genes expressed either before, during or after meiosis, illustrating the specificity of the gene expression program in relation to meiotic progression [13]. In Arabidopsis, pollen development is also transcriptionally modulated and the progression from microspores (post-meiotic cells) to mature pollen is characterized by a large-scale repression of early program genes and the activation of a late gene expression program [14]. The male meiotic cells, the PMCs, share the expression of more than 1.000 orthologous genes with equivalent cells in the mouse and fission yeast [15]. Functional classes like DNA repair and replication were significantly enriched among these conserved genes. This observation shows that some regulatory mechanisms have been 
evolutionarily conserved and that meiosis defines a change in the transcriptional profile of germline development. In fact, in animal spermatogenesis, major transcriptional changes also occur prior to meiotic entry. Studies in mammals and insects point that the pre-meiotic male germline cells activate a specialized transcriptional program [16,17]. These cells produce transcripts that will be required later on (even post-meiotically) for normal cellular development, coding for genes belonging to three major functional classes: metabolism, cytoskeleton and chromosome organization.

Arabidopsis mature pollen grains show a unique transcriptome consisting of 6.587 genes [18]. The enriched mRNAs are related to signalling, cell wall metabolism and cytoskeleton dynamics, reflecting the pollen grain's commitment to germination and tube growth $[18,19]$. This trend is also patent in tobacco, where gene expression profiles during different stages of germination indicate a gradual increase of gametophyte-specific genes, such as regulators of cell tip expansion [20]. Overall, data on pollen transcriptomes have clearly provided important insights into the molecular pathways involved in germ cell delivery. Yet, owing to the profound structural association between germ cells and the somatic vegetative cell, pollen analysis is unsuited for identifying the specificities of the germline transcriptome. This issue can only be satisfactorily addressed via the successful isolation of SCs from the remaining gametophyte. Not surprisingly, the first studies on sperm cell-specific gene expression were performed in plant species where gametes are more accessible for isolation in sufficient amounts, as in the case of Zea mays and Lilium longiflorum [21,22]. These studies provided initial evidence that, unlike previously thought, mature male gametes carry a diverse repertoire of transcripts. Transcriptome studies on growing pollen tubes argue for the existence of de novo transcription at this stage [23,24], but whether this capability is restricted to the VN or is also patent in the male gametes remains to be analysed. In this regard, animal data are less ambiguous, with mature sperm in these species being largely considered transcriptionally silent [25]. The development of a method for SCs isolation in Arabidopsis allowed us to perform the most comprehensive transcriptional study of mature male gametes in this species to date. This led us to the identification of 5.829 sperm cellexpressed genes [26,27]. Out of these, approximately 2.400 showed enriched expression in this particular cell type. The enriched transcripts coded for products involved in DNA repair, ubiquitin-mediated proteolysis and cell cycle progression. These classes are also detected in rice SCs $[28,29]$ and even in human male gametes [30], pointing to the evolutionary conservation of specific molecular processes in the male germline. In Arabidopsis, several male germlinespecific transcripts were also identified, including MALE GAMETE-SPECIFIC HISTONE H3 (MGH3) and HAPLESS2 [HAP2 - also known as GENERATIVE CELLSPECIFIC PROTEIN 1 (GCS1)]. MGH3 is specifically expressed in both the generative cell and the SCs and has a major role in chromatin remodelling [31]. Indeed, both in plants and animals, the chromatin of mature male germ cells is extensively remodelled via the incorporation of germline-specific histone variants (or other chromatin-bound proteins) [32]. Such mechanisms are likely associated with precise structural requirements of chromatin architecture that may or may not associate with gene expression regulation. HAP2(GCS1) encodes a sperm cell-specific surface protein required for pollen tube guidance and gamete fusion [33]. Loss of function of HAP2(GCS1) orthologs in Chlamydomonas 
and Plasmodium also block gamete fusion [34]. Its evolutionary conservation suggests that the functions of $H A P 2(G C S 1)$ in gamete fusion were established in the ancestor of modern plants, protists, and animals [35]. Finally, DUO POLLEN1 (DUO1) is specifically expressed in the male germline soon after PMI and encodes a R2R3 MYB transcription factor [36]. DU01 controls the expression of genes with broad cellular functions such as transcription regulation, protein metabolism and transport [37,38]. Interestingly, among the DU01-regulated genes we can find several male germline determinants such as the aforementioned MGH3 and HAP2(GCS1), and also GAMETE EXPRESSED PROTEIN2 (GEX2), TONOPLAST INTRINSIC PROTEIN 5;1 (TIP5;1) and DUO1ACTIVATED ZINC FINGER1-3 (DAZ1-3). Thus, DU01, by promoting the acquisition of a post-PMI germline transcriptional program, can be considered a major regulator of sperm cell differentiation in plants.

It has been recently shown in plants that transcripts stored in SCs can potentially be delivered upon fertilization and have a role in early embryo development. Such evidence came from the identification of the SHORT SUSPENSOR (SSP) transcripts which accumulate in Arabidopsis SCs but whose encoded protein is only detected in the zygote (hence, after fertilization) [39]. SSP is a protein kinase known to activate the YODA mitogen-activated protein kinase pathway that regulates the first (asymmetric) division of the embryo. In animals, it is also a sperm-borne factor that triggers the onset of embryogenesis. This activation factor has already been identified in mammals and corresponds to the phospholipase C $C$ protein (for a review [40]). Nevertheless, there is no evidence supporting the need for an egg-specific translation of this activating factor. Phospholipase $\mathrm{C} \zeta$ is responsible for the generation of intracellular calcium elevations that ultimately result in egg activation. In vitro fertilization assays in plants have shown that, similar to animals, the fusion between sperm and egg cell also results in changes in intracellular calcium levels. However, in plants this mechanism is insufficient for egg activation [41,42], suggesting that the onset of embryogenesis is dependent on additional factors. RNAs that accumulate in SCs (such as those for SSP) may be one of these factors. Could animal sperm-derived RNAs play a role in embryo development, as speculated in plants? Certainly, there is ample opportunity for such a mechanism: mature mammalian sperm contain a diverse RNA population, comprising not only mRNAs but also different classes of small RNAs such as miRNAs and Piwi-interacting RNAs (piRNAs) $[43,44]$. Nevertheless, the successful cloning of animals using somatic cell nuclear transfer provides proof of concept that these sperm molecules are not essential for development to term. It should be noted that the low success rate of animal cloning procedures does not preclude a modulating role for spermderived RNAs during the initial stages of embryo development, as previously suggested [45].

Small RNA-mediated epigenetic regulation of the male germline

Recently, a complex repertoire of small RNAs has been detected in mature male gametes of both plants and animals. Given their role in the modulation of gene expression, it is tempting to speculate that they may serve instrumental roles in gametogenesis and in early post-fertilization development. Small RNAs are abundant in the pollen of Arabidopsis and rice and can be divided in two major 
classes: microRNAs (miRNAs) [46,47] and short interfering RNAs (siRNAs) [4850]. Both serve as regulators of gene expression, either transcriptionally or posttranscriptionally.

miRNAs are single-stranded molecules, usually 21 to 22 nucleotides long, that although synthesized by specific genes can regulate the translation of mRNAs derived from several others. They are initially produced in the form of larger RNA precursors, which are later processed (in plants by DICER-LIKE1, DCL1) to yield mature miRNAs. These molecules are then recruited by an AGO protein (part of the RNA-induced silencing complex - RISC) and are guided to their mRNA targets to initiate endonucleolytic cleavage or translational inhibition [51]. Studies have shown not only that miRNAs are abundant in pollen, but also that their precursors and target cleavage products can be detected [52]. In isolated Arabidopsis SCs, miRNAs belonging to 83 different families could be identified [46], with recent studies using artificial constructs indicating that, at least in male gametes and like what was seen in animals, these molecules may also act through translational repression [53]. Similarly, miRNAs are abundantly expressed during animal spermatogenesis, where they play important roles in the spatio-temporal regulation of gene expression (for a review [54]). It should be noted that unlike in mammals, where miRNAs have been shown to play key roles in early male germ cell proliferation and late spermatogenesis, in plants the putative developmental relevance of any natural miRNA-target pair predicted to exist in the male gametophyte lacks experimental validation. In this context it is also intriguing that $d c l 1$ heterozygous mutant plants (in which half of the pollen do not express DCL1), have no discernible male transmission deficiencies [55].

siRNAs occur naturally in plants and lower animals, while their occurrence in mammals is still controversial. In plants, siRNAs originate from double stranded RNAs before being processed into 19 to 25 nucleotides long siRNAs. These molecules are instrumental for the reprogramming of the male germline, acting in the silencing of transposable elements (TEs). In Arabidopsis certain TEs are reactivated in the $\mathrm{VN}$, coincident with the loss of DECREASE IN DNA METHYLATION 1 (DDM1) enzyme, and resulting in the production of siRNAs by the VN. In a manner still to be clarified, these siRNAs accumulate in the neighboring SCs, thus ensuring TEs silencing in the germline [48]. An equivalent mechanism is also present in animals, where piRNAs have homologous functions to those of plant siRNAs [56,57]. More specifically, the piRNA pathway has been shown to be required to repress TEs at two developmental stages: i- during spermatogenesis, via post-transcriptional regulation; and ii- during germline reprogramming in embryogenesis, by purportedly directing RNA-dependent DNA methylation in developing fetal germ cells [58,59]. Overall, small RNA mechanisms seem to have been evolutionarily-conserved as means of safeguarding the (epi)genetic information contained in male gametes against possible disruptions caused by mobile genomic elements.

\section{Conclusions and future perspectives}

It can be argued that sexual reproduction is, ultimately, defined by the capability of germline cells (or their precursors) to undergo meiosis. Meiosis, besides ensuring DNA recombination and reductive chromosome segregation, dictates profound changes in the germline gene expression program. These changes are 
particularly evident when comparing disparate Eukaryotic species and suggest that male gametogenesis implies the successful orchestration of two evolutionarily-conserved gene expression programs: one pre-meiotic, the other post-meiotic. We can speculate that the first program commits cells to meiosis while the second ensures the acquisition of the fertilizing ability. Such ability is manifested by two equally conserved hallmarks: extensive chromatin remodeling and exposure of cell surface proteins required for subsequent fusion with the female gamete. Regulation via small RNAs adds an extra layer of complexity to both expression programs, with these molecules playing key roles in the silencing of mobile genomic elements and, perhaps, on the actual regulation of the differentiation program.

In light of the rather limited success of candidate gene-driven and highthroughput platforms in identifying novel regulators of germline function, our understanding of basic mechanisms of male germ cell development may benefit from an evolutionary developmental biology approach. The integrated outlook provided by such approach will increase the likelihood of identifying novel, evolutionarily-conserved molecular mechanisms of germline development.

Acknowledgements

We thank Leonor Boavida for critical reading of the manuscript.

Funding

JDB acknowledges Fundação para a Ciência e a Tecnologia (FCT), Portugal, for research grant [PTDC/BIA-BCM/103787/2008]. RGM acknowledges FCT for [PTDC/SAU-BID/111796/2009] and [PTDC/BIA-BCM/111822/2009]. PAP is supported by a FCT PhD fellowship [SFRH/BD/63477/2009] and PNC is supported by a FCT Post-Doctoral fellowship [SFRH/BPD/84214/2012].

\section{References}

1 Extavour, C.G. and Akam, M. (2003) Mechanisms of germ cell specification across the metazoans: epigenesis and preformation. Development 130, 5869-5884

2 Boavida, L.C., Becker, J.D. and Feijó, J.A. (2005) The making of gametes in higher plants. Int. J. Dev. Biol. 49, 595-614

3 Chen, Y.C. and McCormick, S. (1996) sidecar pollen, an Arabidopsis thaliana male gametophytic mutant with aberrant cell divisions during pollen development. Development 122, 3243-3253

4 Park, S.K., Howden, R. and Twell, D. (1998) The Arabidopsis thaliana gametophytic mutation gemini pollen1 disrupts microspore polarity, division asymmetry and pollen cell fate. Development 125, 3789-3799 
5 Park, S.K. and Twell, D. (2001) Novel patterns of ectopic cell plate growth and lipid body distribution in the Arabidopsis gemini pollen1 mutant. Plant Physiol. 126, 899-909

6 McCue, A.D., Cresti, M., Feijó, J.A. and Slotkin, R.K. (2011) Cytoplasmic connection of sperm cells to the pollen vegetative cell nucleus : potential roles of the male germ unit revisited. J. Exp. Bot. 62, 1621-1631

7 Lalanne, E. and Twell, D. (2002) Genetic Control of Male Germ Unit Organization in Arabidopsis. Plant Physiol. 129, 865-875

8 Boavida, L.C., Qin, P., Broz, M., Becker, J.D. and McCormick, S. (2013) Arabidopsis tetraspanins are confined to discrete expression domains and cell types in reproductive tissues and form homo- and heterodimers when expressed in yeast. Plant Physiol. 163, 696-712

9 Lesch, B.J. and Page, D.C. (2012) Genetics of germ cell development. Nat. Rev. Genet. 13, 781-794

10 Guo, G.Q. and Zheng, G.C. (2004) Hypotheses for the functions of intercellular bridges in male germ cell development and its cellular mechanisms. J. Theor. Biol. 229, 139-146

11 Ward, S., Argon, Y. and Nelson, G.A. (1981) Sperm morphogenesis in wildtype and fertilization-defective mutants of Caenorhabditis elegans. J. Cell Biol. 91, 26-44

12 Skinner, M.K. (1991) Cell-cell interactions in the testis. Endocr. Rev. 12, 45-77

13 Ma, J., Skibbe, D.S., Fernandes, J. and Walbot, V. (2008) Male reproductive development: gene expression profiling of maize anther and pollen ontogeny. Genome Biol. 9, R181

14 Honys, D. and Twell, D. (2004) Transcriptome analysis of haploid male gametophyte development in Arabidopsis. Genome Biol. 5, R85

15 Yang, H., Lu, P., Wang, Y. and Ma, H. (2011) The transcriptome landscape of Arabidopsis male meiocytes from high-throughput sequencing: the complexity and evolution of the meiotic process. Plant J. 65, 503-516

16 Schultz, N., Hamra, F.K. and Garbers, D.L. (2003) A multitude of genes expressed solely in meiotic or postmeiotic spermatogenic cells offers a myriad of contraceptive targets. Proc. Natl. Acad. Sci. U.S.A. 100, 1220112206

17 White-Cooper, H. (2010) Molecular mechanisms of gene regulation during Drosophila spermatogenesis. Reproduction 139, 11-21 
18 Pina, C., Pinto, F., Feijó, J.A. and Becker, J.D. (2005) Gene Family Analysis of the Arabidopsis Pollen Transcriptome Reveals Biological Implications for Cell Growth, Division Control, and Gene Expression Regulation. Plant Physiol. 138, 744-756

19 Becker, J.D., Boavida, L.C., Carneiro, J., Haury, M. and Feijó, J.A. (2003) Transcriptional Profiling of Arabidopsis Tissues Reveals the Unique Characteristics of the Pollen Transcriptome. Plant Physiol. 133, 713-725

20 Hafidh, S., Breznenová, K., Růžička, P., Feciková, J., Capková, V. and Honys, D. (2012) Comprehensive analysis of tobacco pollen transcriptome unveils common pathways in polar cell expansion and underlying heterochronic shift during spermatogenesis. BMC Plant Biol. 12, 24

21 Engel, M.L., Chaboud, A., Dumas, C. and McCormick, S. (2003) Sperm cells of Zea mays have a complex complement of mRNAs. Plant J. 34, 697-707

22 Okada, T., Bhalla, P.L. and Singh, M.B. (2006) Expressed sequence tag analysis of Lilium longiflorum generative cells. Plant Cell Physiol. 47, 698705

23 Qin, Y., Leydon, A.R., Manziello, A., Pandey, R., Mount, D., Denic, S., Vasic, B., Johnson, M.A. and Palanivelu, R. (2009) Penetration of the stigma and style elicits a novel transcriptome in pollen tubes, pointing to genes critical for growth in a pistil. PLoS Genet. 5, e1000621

24 Boavida, L.C., Borges, F., Becker, J.D. and Feijó, J.A. (2011) Whole genome analysis of gene expression reveals coordinated activation of signaling and metabolic pathways during pollen-pistil interactions in Arabidopsis. Plant Physiol. 155, 2066-2080

25 White-Cooper, H. and Davidson, I. (2011) Unique aspects of transcription regulation in male germ cells. Cold Spring Harb. Perspect. Biol. 3, a002626

26 Borges, F., Gomes, G., Gardner, R., Moreno, N., Mccormick, S., Feijó J.A. and Becker, J.D. (2008) Comparative transcriptomics of Arabidopsis sperm cells. Plant Physiol. 148, 1168-1181

27 Borges, F., Gardner, R., Lopes, T., Calarco, J.P., Boavida, L.C., Slotkin, R.K., Martienssen, R.A. and Becker, J.D. (2012) FACS-based purification of Arabidopsis microspores, sperm cells and vegetative nuclei. Plant Methods 8, 44

28 Anderson, S.N., Johnson, C.S., Jones, D.S., Conrad, L.J., Gou, X., Russell, S.D. and Sundaresan, V. (2013) Transcriptomes of isolated Oryza sativa gametes characterized by deep sequencing: evidence for distinct sexdependent chromatin and epigenetic states before fertilization. Plant J. Advance online publication, doi: 10.1111/tpj.12336 
29 Russell, S.D., Gou, X., Wong, C.E., Wang, X., Yuan, T., Wei, X., Bhalla, P.L. and Singh, M.B. (2012) Genomic profiling of rice sperm cell transcripts reveals conserved and distinct elements in the flowering plant male germ lineage. New Phytol. 195, 560-573

30 Wuest, S.E., Vijverberg, K., Schmidt, A., Weiss, M., Gheyselinck, J., Lohr, M., Wellmer, F., Rahnenführer, J., von Mering, C. and Grossniklaus, U. (2010) Arabidopsis female gametophyte gene expression map reveals similarities between plant and animal gametes. Curr. Biol. 20, 506-512

31 Okada, T., Endo, M., Singh, M.B. and Bhalla, P.L. (2005) Analysis of the histone $\mathrm{H} 3$ gene family in Arabidopsis and identification of the malegamete-specific variant AtMGH3. Plant J. 44, 557-568

32 Carrell, D.T. (2012) Epigenetics of the male gamete. Fertil. Steril. 97, 267274

33 von Besser, K., Frank, A.C., Johnson, M.A. and Preuss, D. (2006) Arabidopsis HAP2 (GCS1) is a sperm-specific gene required for pollen tube guidance and fertilization. Development 133, 4761-4769

34 Liu, Y., Tewari, R., Ning, J., Blagborough, A.M., Garbom, S., Pei, J., Grishin, N. V., Steele, R.E., Sinden, R.E., Snell, W.J. and Billker, O. (2008) The conserved plant sterility gene HAP2 functions after attachment of fusogenic membranes in Chlamydomonas and Plasmodium gametes. Genes Dev. 22, 1051-1068

35 Steele, R.E. and Dana, C.E. (2009) Evolutionary history of the HAP2/GCS1 gene and sexual reproduction in metazoans. PLoS One 4, e7680

36 Rotman, N., Durbarry, A., Wardle, A., Yang, W.C., Chaboud, A., Faure, J.E., Berger, F. and Twell, D. (2005) A novel class of MYB factors controls sperm-cell formation in plants. Curr. Biol. 15, 244-248

37 Brownfield, L., Hafidh, S., Borg, M., Sidorova, A., Mori, T. and Twell, D. (2009) A plant germline-specific integrator of sperm specification and cell cycle progression. PLoS Genet. 5, e1000430

38 Borg, M., Brownfield, L., Khatab, H., Sidorova, A., Lingaya, M. and Twell, D. (2011) The R2R3 MYB transcription factor DUO1 activates a male germline-specific regulon essential for sperm cell differentiation in Arabidopsis. Plant Cell 23, 534-549

39 Bayer, M., Nawy, T., Giglione, C., Galli, M., Meinnel, T. and Lukowitz, W. (2009) Paternal Control of Embryonic Patterning in Arabidopsis thaliana. Science 323, 1485-1488

40 Clift, D. and Schuh, M. (2013) Restarting life: fertilization and the transition from meiosis to mitosis. Nat. Rev. Mol. Cell Biol. 14, 549-562 
41 Antoine, A.F., Faure, J.E., Cordeiro, S., Dumas, C., Rougier, M. and Feijó, J.A. (2000) A calcium influx is triggered and propagates in the zygote as a wavefront during in vitro fertilization of flowering plants. Proc. Natl. Acad. Sci. U.S.A. 97, 10643-10648

42 Antoine, A.F., Faure, J.E., Dumas, C. and Feijó, J.A. (2001) Differential contribution of cytoplasmic $\mathrm{Ca} 2+$ and $\mathrm{Ca} 2+$ influx to gamete fusion and egg activation in maize. Nat. Cell Biol. 3, 1120-1123

43 Ostermeier, G.C., Goodrich, R.J., Moldenhauer, J.S., Diamond, M.P. and Krawetz, S.A. (2005) A suite of novel human spermatozoal RNAs. J. Androl. 26, 70-74

44 Krawetz, S.A., Kruger, A., Lalancette, C., Tagett, R., Anton, E., Draghici, S. and Diamond, M.P. (2011) A survey of small RNAs in human sperm. Hum. Reprod. 26, 3401-3412

45 Liu, W.M., Pang, R.T., Chiu, P.C., Wong, B.P., Lao, K., Lee, K.F. and Yeung, W.S. (2012) Sperm-borne microRNA-34c is required for the first cleavage division in mouse. Proc. Natl. Acad. Sci. U.S.A. 109, 490-494

46 Borges, F., Pereira, P.A., Slotkin, R.K., Martienssen, R.A. and Becker, J.D. (2011) MicroRNA activity in the Arabidopsis male germline. J. Exp. Bot. 62, $1611-1620$

47 Wei, L.Q., Yan, L.F. and Wang, T. (2011) Deep sequencing on genome-wide scale reveals the unique composition and expression patterns of microRNAs in developing pollen of Oryza sativa. Genome Biol. 12, R53

48 Slotkin, R.K., Vaughn, M., Borges, F., Tanurdzić, M., Becker, J.D., Feijó, J.A and Martienssen, R.A. (2009) Epigenetic reprogramming and small RNA silencing of transposable elements in pollen. Cell 136, 461-472

49 Le Trionnaire, G., Grant-Downton, R.T., Kourmpetli, S., Dickinson, H.G. and Twell, D. (2011) Small RNA activity and function in angiosperm gametophytes. J. Exp. Bot. 62, 1601-1610

50 Nobuta, K., Venu, R.C., Lu, C., Beló, A., Vemaraju, K., Kulkarni, K., Wang, W., Pillay, M., Green, P.J., Wang, G.L. and Meyers, B.C. (2007) An expression atlas of rice mRNAs and small RNAs. Nat. Biotechnol. 25, 473-477

51 Rogers, K. and Chen, X. (2013) Biogenesis, turnover, and mode of action of plant microRNAs. Plant Cell 25, 2383-2399

52 Grant-Downton, R., Hafidh, S., Twell, D. and Dickinson, H.G. (2009) Small RNA Pathways Are Present and Functional in the Angiosperm Male Gametophyte. Mol. Plant 2, 500-512 
53 Grant-Downton, R., Kourmpetli, S., Hafidh, S., Khatab, H., Le Trionnaire, G., Dickinson, H. and Twell, D. (2013) Artificial microRNAs reveal cell-specific differences in small RNA activity in pollen. Curr. Biol. 23, 599-601

54 McIver, S.C., Roman, S.D., Nixon, B. and McLaughlin, E.A. (2012) miRNA and mammalian male germ cells. Hum. Reprod. Update 18, 44-59

55 Nodine, M.D. and Bartel, D.P. (2010) MicroRNAs prevent precocious gene expression and enable pattern formation during plant embryogenesis. Genes Dev. 24, 2678-2692

56 Di Giacomo, M., Comazzetto, S., Saini, H., De Fazio, S., Carrieri, C., Morgan, M., Vasiliauskaite, L., Benes, V., Enright, A.J. and O'Carroll, D. (2013) Multiple Epigenetic Mechanisms and the piRNA Pathway Enforce LINE1 Silencing during Adult Spermatogenesis. Mol. Cell 50, 601-608

57 Aravin, A.A., Sachidanandam, R., Bourc'his, D., Schaefer, C., Pezic, D., Toth, K.F., Bestor, T. and Hannon, G.J. (2008) A piRNA pathway primed by individual transposons is linked to de novo DNA methylation in mice. Mol. Cell 31, 785-799

58 Kuramochi-Miyagawa, S., Watanabe, T., Gotoh, K., Totoki, Y., Toyoda, A., Ikawa, M., Asada, N., Kojima, K., Yamaguchi, Y., Ijiri, T.W., Hata, K., Li, E., Matsuda, Y., Kimura, T., Okabe, M., Sakaki, Y., Sasaki, H. and Nakano, T. (2008) DNA methylation of retrotransposon genes is regulated by Piwi family members MILI and MIWI2 in murine fetal testes. Genes Dev. 22, 908-917

59 Aravin, A.A., Hannon, G.J. and Brennecke, J. (2007) The Piwi-piRNA Pathway Provides an Adaptive Defense in the Transposon Arms Race. Science 318, 761-764

Figure legend

Figure 1. Unifying principles of male germline establishment and development. Male germline development in higher plants is depicted, using Arabidopsis thaliana as model. Text boxes refer to common principles of male germline development between plants and animals. PSC, primary sporogenous cell; UNM, uninucleate microspore; BCP, bicellular pollen; MPG, mature pollen grain; PMI, pollen mitosis I; PMII, pollen mitosis II; CC, cytomictic channel; GC, generative cell; VE, vegetative endomembrane; SCs, sperm cells. 
Figure 1

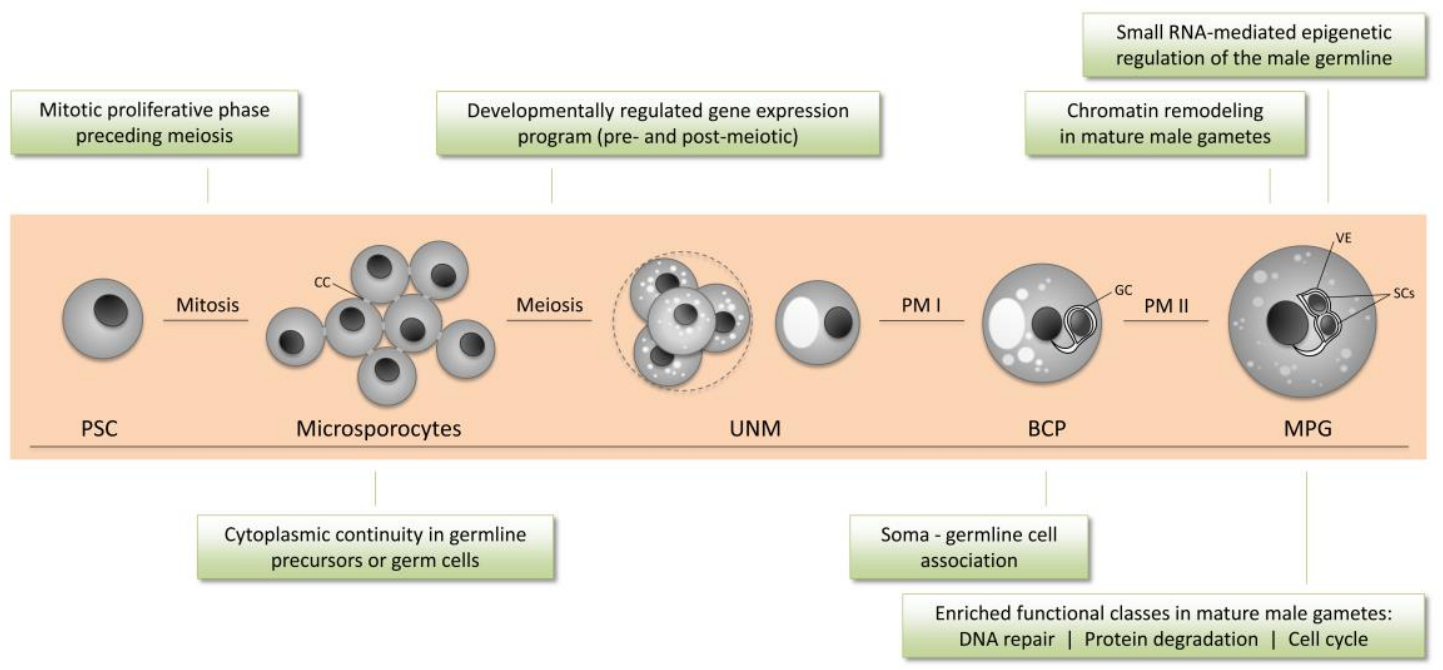

